\title{
Cine-Magnetic Resonance Imaging Evaluation of Communication between Middle Cranial Fossa Arachnoid Cysts and Cisterns
}

\author{
Takahiko EgUCHI, Toshiaki TAOKA*, Yuji NIKAIDO, Kazuaki SHIOMI, \\ Takatoshi FuJIMOTO, Hiroyuki OTSUKA, and Hiroshi TAKEUCHI
}

\author{
Department of Neurosurgery, Osaka-Minami National Hospital, Kawachinagano, Osaka; \\ *Department of Radiology, Nara Prefectural Hospital, Nara
}

\begin{abstract}
Cine-magnetic resonance (MR) imaging examinations were performed in 10 patients with middle cranial fossa arachnoid cysts to evaluate communication between the cysts and the normal cerebrospinal fluid (CSF) space. Eight of 10 patients were evaluated by time of flight cine-MR imaging, and two by phase contrast cine-MR imaging. Two patients underwent membranectomy of the cysts, and were evaluated both pre- and postoperatively. Computed tomography cisternography was used to confirm communication between the cysts and the surrounding cisterns. Pulsatile fluid motion within the cysts was present in all patients. However, marked fluid motion and jet flow between the cysts and the surrounding cisterns were only observed in communicating cysts. In the two patients who underwent membranectomy, postoperative examination found greater fluid motion and jet flow not previously present. Cine-MR imaging demonstration of marked pulsatile fluid motion accompanied by jet flow suggests that a cyst communicates with the normal CSF space.
\end{abstract}

Key words: cine-magnetic resonance imaging, arachnoid cyst, examination

\section{Introduction}

Arachnoid cysts are a common condition consisting of fluid-filled cavities of uncertain origin. Treatment of such cysts requires assessment of the communication between the cysts and the surrounding normal cerebrospinal fluid (CSF) space. ${ }^{5)}$ Computed tomography (CT) cisternography clearly demonstrates the relationship between the cysts and the physiological CSF space, but is rather invasive. Magnetic resonance (MR) imaging is sensitive to slow CSF flow and therefore can demonstrate flow voids due to pulsatile motion of CSF. ${ }^{2,3,6,10)}$ Recently, noninvasive cine-MR imaging has been used to evaluate CSF flow in disorders such as normal-pressure hydrocephalus. ${ }^{7}$

This study used cine-MR imaging to assess pulsatile fluid motion in 10 patients with middle cranial fossa arachnoid cysts to evaluate the communication between the cysts and the surrounding cisterns.

Received August 17, 1995; Accepted January 11, 1996

\section{Materials and Methods}

Ten patients, seven males and three females (aged 4$65 \mathrm{yrs}$ ), with middle fossa arachnoid cysts were evaluated using cine-MR imaging between August 1992 and December 1994 (Table 1). Five patients were asymptomatic, three had headache, one had exophthalmos, and one had a cranial deformity. The cysts were divided into three groups according to size: three small cysts located at the anterior pole of the middle cranial fossa, three medium-sized cysts which occupied the anterior half of the middle cranial fossa, and four large cysts which occupied almost the entire middle cranial fossa. CT cisternography was performed in all patients. Communication between the cyst and the cistern was defined as contrast medium present in the cyst and the surrounding cistern on a CT scan obtained 3 hours following injection (rapid filling). Two patients underwent membranectomy with craniotomy and were assessed both preoperatively and postoperatively.

The first eight patients of this series were evaluated by time of flight (TOF) cine-MR imaging (Magnetom 
Table 1 Summary of cases

\begin{tabular}{|c|c|c|c|c|c|c|}
\hline \multirow{2}{*}{ Case No. } & \multirow{2}{*}{ Age/Sex } & \multirow{2}{*}{ Size } & \multirow{2}{*}{ Symptom } & \multirow{2}{*}{$\begin{array}{l}\text { Communication } \\
\text { with CSF space }\end{array}$} & \multicolumn{2}{|c|}{ Cine-MR imaging findings } \\
\hline & & & & & Pulsatile fluid motion & Jet flow \\
\hline 1 & $64 / F$ & small & asymptomatic & + & moderate & - \\
\hline 2 & $34 / F$ & small & asymptomatic & + & marked & + \\
\hline 3 & $20 / \mathrm{M}$ & small & exophthalmos & - & slight/moderate* & $-t^{*}$ \\
\hline 4 & $31 / \mathrm{M}$ & medium & asymptomatic & - & moderate & - \\
\hline 5 & $4 / M$ & medium & asymptomatic & - & moderate & - \\
\hline 6 & $10 / \mathrm{M}$ & medium & asymptomatic & - & slight & - \\
\hline 7 & $60 / \mathrm{M}$ & large & headache & - & slight & - \\
\hline 8 & $65 / \mathrm{M}$ & large & headache & - & slight & - \\
\hline 9 & $26 / \mathrm{F}$ & large & headache & - & slight/marked ${ }^{*}$ & $-1+*$ \\
\hline 10 & $8 / \mathrm{M}$ & large & skull deformity & - & slight & - \\
\hline
\end{tabular}

*Before/after membranectomy.

Impact 1.0 T; Siemens Aktiengesellschaft, Erlangen, Bayern, Germany) and the last two patients were evaluated by phase contrast (PC) cine-MR imaging (Signa 1.5 T; General Electric Medical System, Milwaukee, Wis., U.S.A.). The parameters for the TOF series were FLASH 2 D/flip angle $20^{\circ} /$ repetition time (TR) $50 \mathrm{msec} /$ echo time (TE) $15-20 \mathrm{msec} /$ cardiac gating, and for the PC series TR $30 \mathrm{msec} /$ TE $11 \mathrm{msec} /$ flip angle $30^{\circ} /$ velocity, encoding $15-20$ $\mathrm{msec} /$ peripheral pulse gating. Slice thickness was 5 $\mathrm{mm}$ for each sequence. Axial, coronal, and sagittal images were obtained of each patient.

\section{Results}

Cine-MR imaging identified pulsatile fluid motion within the arachnoid cysts in all patients (Table 1).

One of two small communicating cysts showed moderate pulsatile fluid motion within the cyst. The other small cyst had marked pulsatile fluid motion within the cyst and jet flow between the cyst and the suprasellar cistern (Fig. 1). The third small cyst was noncommunicating and was treated by membranectomy. Preoperative cine-MR imaging demonstrated slight pulsatile fluid motion within the cyst (Fig. 2). Postoperative examination demonstrated moderate fluid motion and jet flow between the cyst and the suprasellar cistern (Fig. 3).

The three medium-sized cysts were all noncommunicating. The pulsatile movement within the cysts was moderate in two cases and slight in one. No jet flow to the surrounding cisterns were identified in any case.

The four large cysts were all noncommunicating. The fluid motion within the large cysts was slight in all cases (Fig. 4). One patient underwent membranec-
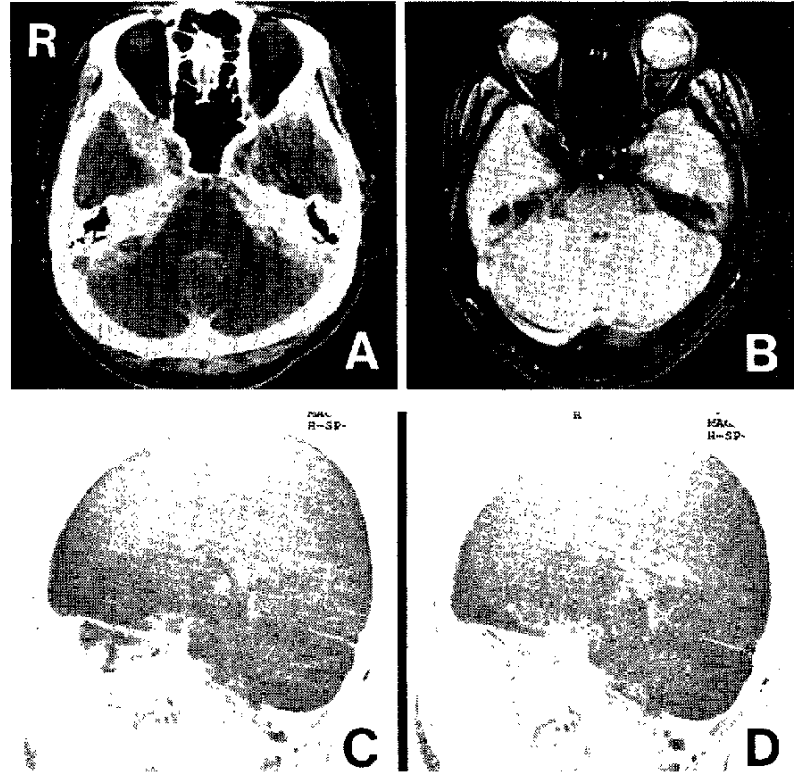

Fig. 1 Case 2. CT cisternogram showing rapid filling of contrast medium (A). TOF cine-MR images showing marked pulsatile fluid motion within the cyst (B-D), and jet flow between the cysts and suprasellar cistern (C, D).

tomy. Postoperative cine-MR imaging showed marked fluid motion within the cyst and jet flow between the cyst and the surrounding cistern (Figs. 5 and 6).

Marked fluid motion and jet flow between the cysts and the cisterns were found in only communicating cysts, while only slight fluid motion was found in isolated cysts. 

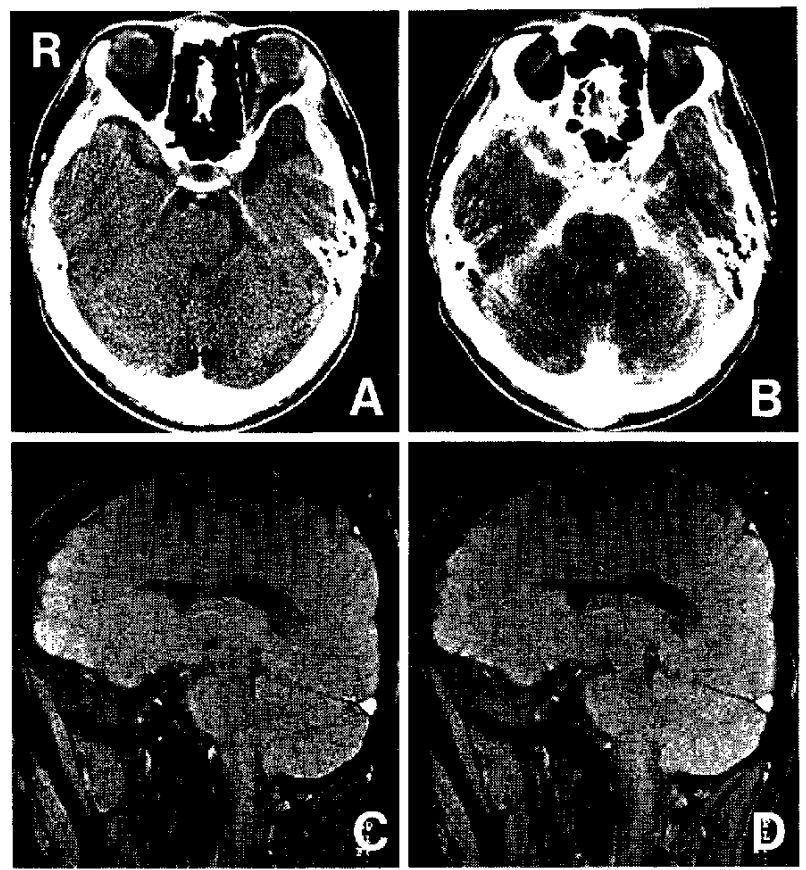

Fig. 2 Case 3 (preoperative study). CT scan showing a small arachnoid cyst at the left anterior middle cranial fossa (A). CT cisternogram showing poor filling of contrast medium within the cyst (B). TOF cine-MR images showing slight pulsatile fluid motion within the cyst $(\mathrm{C}$, D).

\section{Discussion}

Previously, the steady state free procession MR technique which is sensitive to slow flow has been used to evaluate intracranial arachnoid cysts. ${ }^{3)}$ Irregular signal loss consistent with fluid motion was present in isolated cysts as well as physiological CSF spaces. The forces transmitted from the surrounding pulsating brain parenchyma to the cysts presumably created the pulsatile fluid motion within the isolated cysts. Therefore, the authors concluded that invasive cisternography was required to evaluate these cystic lesions. However, their conclusions were based on static images depending on the flow void effect, taken of only three isolated arachnoid cysts of which one was a middle cranial fossa cyst. Our study also found fluid motion within all middle fossa arachnoid cysts. However, the degree of fluid motion in the communicating cysts was greater than in the isolated cysts. Furthermore, the fluid motion in the postoperative cysts was greater than in preoperative studies.

The driving force causing the CSF motion could
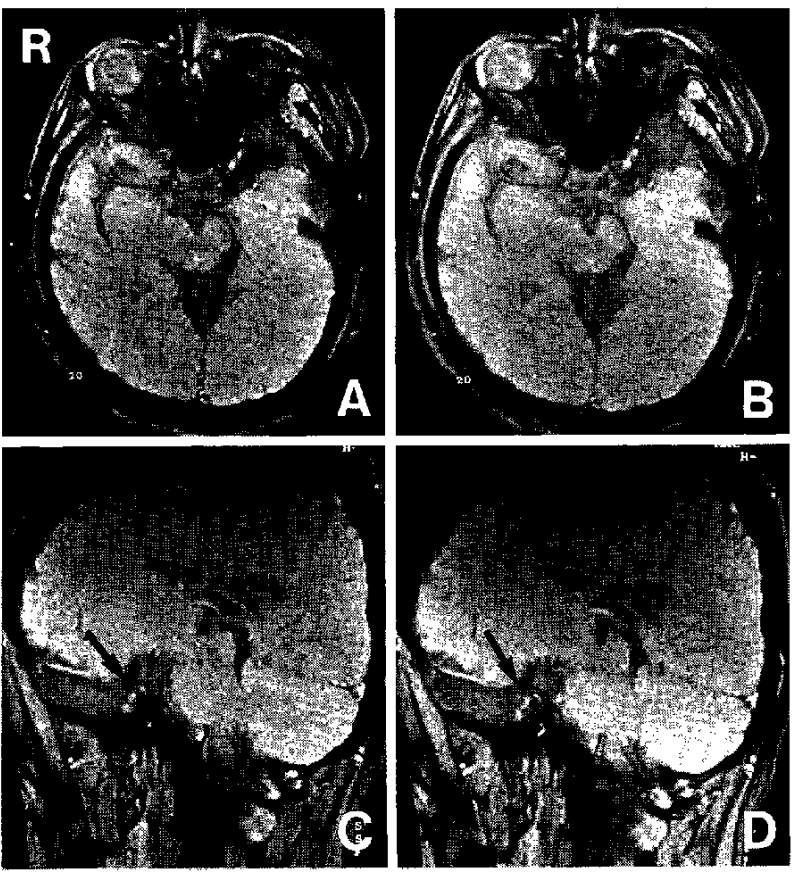

Fig. 3 Case 3 (postoperative study). TOF cine-MR images showing greater pulsatile fluid motion than in the preoperative study (A-D), and the jet flow from the suprasellar cistern into the cyst (C, D; arrows).

easily be transmitted to fluid collections, because the force originates from arterial pulsation ${ }^{1)}$ and parenchymal pulsation $^{4)}$ which are adjacent to the cysts. The amplitude of to-and-fro CSF motion is affected by several factors. Intracranial compliance affects the degree of pulsatile CSF motion. ${ }^{8}$ The velocity of normal CSF measured by PC cine-MR imaging in small cisterns is faster than in large cisterns. ${ }^{9)}$ Presumably the velocity of the CSF flow is affected by factors such as the anatomical size of the structure through which the CSF passes, and varies from patient to patient. In our preoperative examinations, the cysts communicating to the normal CSF spaces showed greater pulsatile fluid motion. However, these communicating cysts were all small, while most isolated cysts were large. The difference in the size of cysts might affect the amplitude of fluid motion, so we doubt whether the communication between arachnoid cysts and cisterns can be evaluated using the amplitude of fluid motion, although the communication with cisterns affects fluid motion. In contrast to the amplitude of fluid motion, jet flow between an arachnoid cyst and the surrounding cistern confirmed the communication between the cyst and subarachnoid space. 

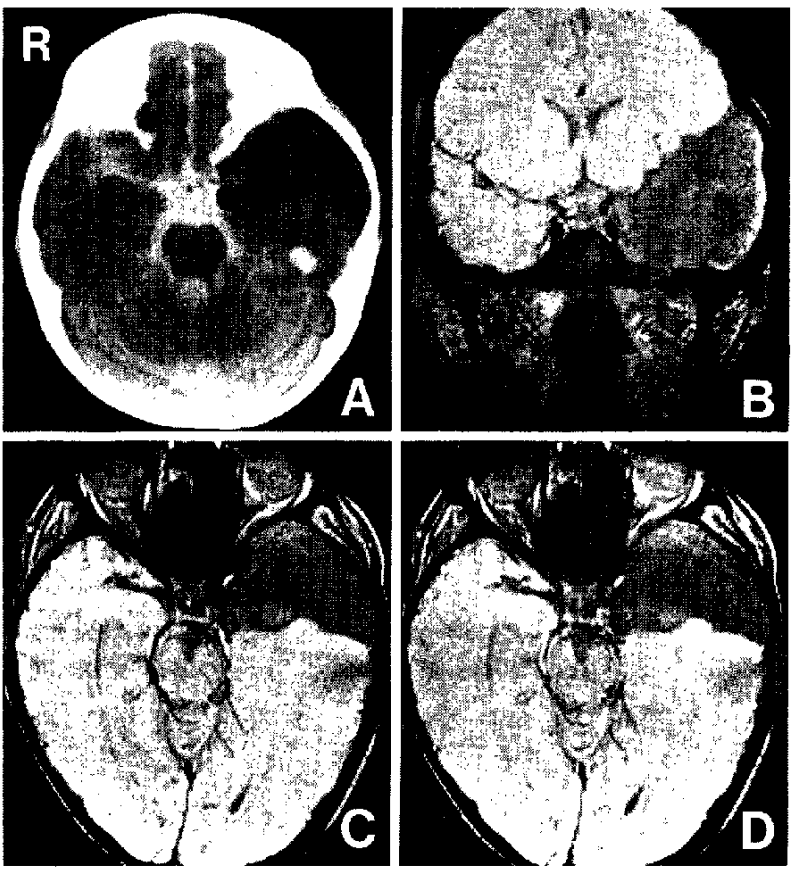

Fig. 4 Case 10. CT cisternogram showing no filling of contrast medium (A). TOF cine-MR images showing slight pulsatile fluid movement within the cyst (B-D).

We used both PC and TOF cine techniques in this study. In general, the intensity of MR image is proportional to the velocity of CSF with the PC technique, while the intensity is affected not only by the velocity, but also the inflow effect and phasedephase effect in the TOF technique. Thus, we conclude the $\mathrm{PC}$ cine technique is a more reliable in- dicator of CSF flow velocity.

Cine-MR imaging is useful for the noninvasive evaluation of middle cranial fossa arachnoid cysts. Marked fluid motion within a middle cranial fossa arachnoid cyst suggests that the cyst communicates with the normal CSF space. Jet flow confirms that the cyst communicates with the cisterns.

\section{References}

1) Adolph RJ, Fukumi H, Flowler NO: Origin of cerebrospinal fluid pulsations. $A m J$ Physiol 212: 840-846, 1965

2) Bradley WG Jr, Kortman KE, Burugonyne B: Flowing cerebrospinal fluid in normal and hydrocephalic states, appearance on MR images. Radiology 159: 611,1985

3) Brooks ML, Jolesz FA, Patz S: MRI of pulsatile CSF motion within arachnoid cysts. Magn Reson Imaging 6: 575-584, 1988

4) Feinberg DA, Mark AS: Human brain motion and cerebrospinal fluid circulation demonstrating MR velocity imaging. Radiology 163: 793-799, 1987

5) Galassi E, Tognetti F, Gaist G, Fagioli L, Frank F, Frank G: CT scan and metrizamide CT cisternography in arachnoid cysts in the middle cranial fossa. Classification and pathophysiological aspect. Surg Neurol 17: 363-369, 1982

6) Le Bihan D, Breton E, Lallemand D, Grenier P, Cabanis E, Laval-Jeanet M: MR imaging of intravoxel incoherent motions: Application to diffusion and perfusion in neurologic disorders. Radiology 161: 401-407, 1986

7) Mabe $H$, Nagai $H$, Banno $T$ : An analysis of the cerebrospinal fluid dynamics in patients with normal pressure hydrocephalus. No Shinkei Geka Journal 3: 319-323, 1994 (in Japanese)
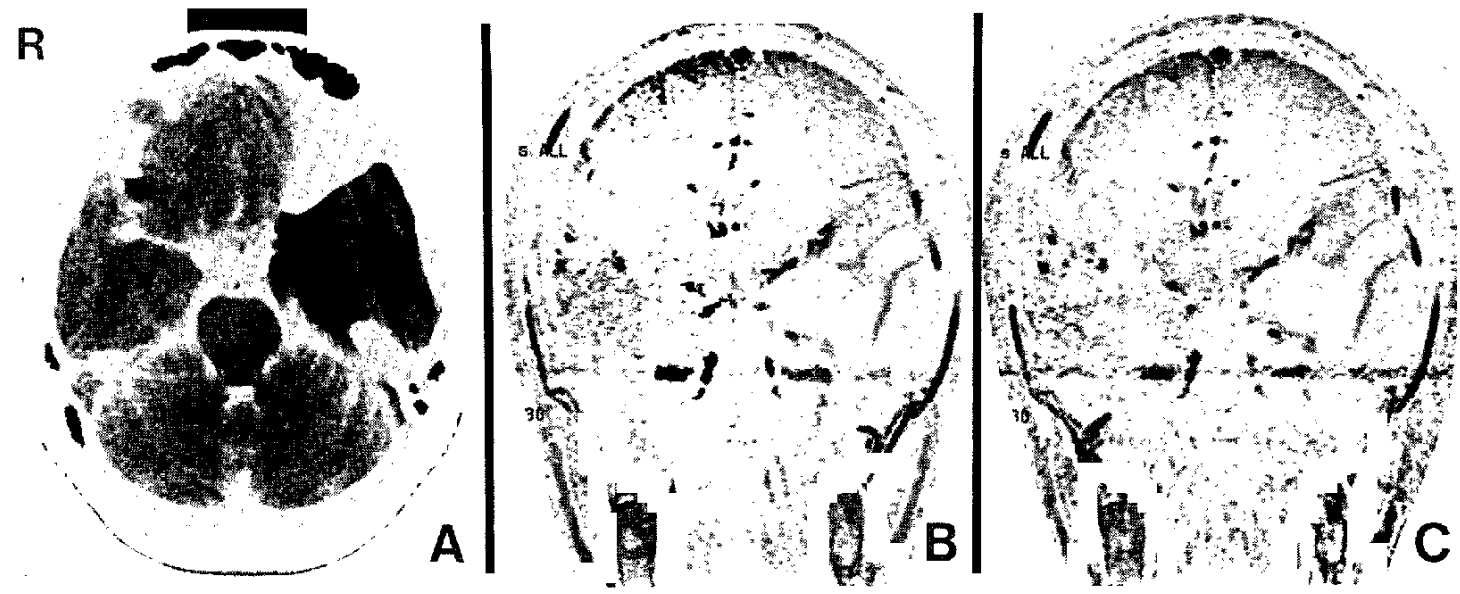

Fig. 5 Case 9 (preoperative study). CT cisternogram revealing poor filling of contrast medium (A). PC cine-MR images showing slight pulsatile fluid motion $(B, C)$. 

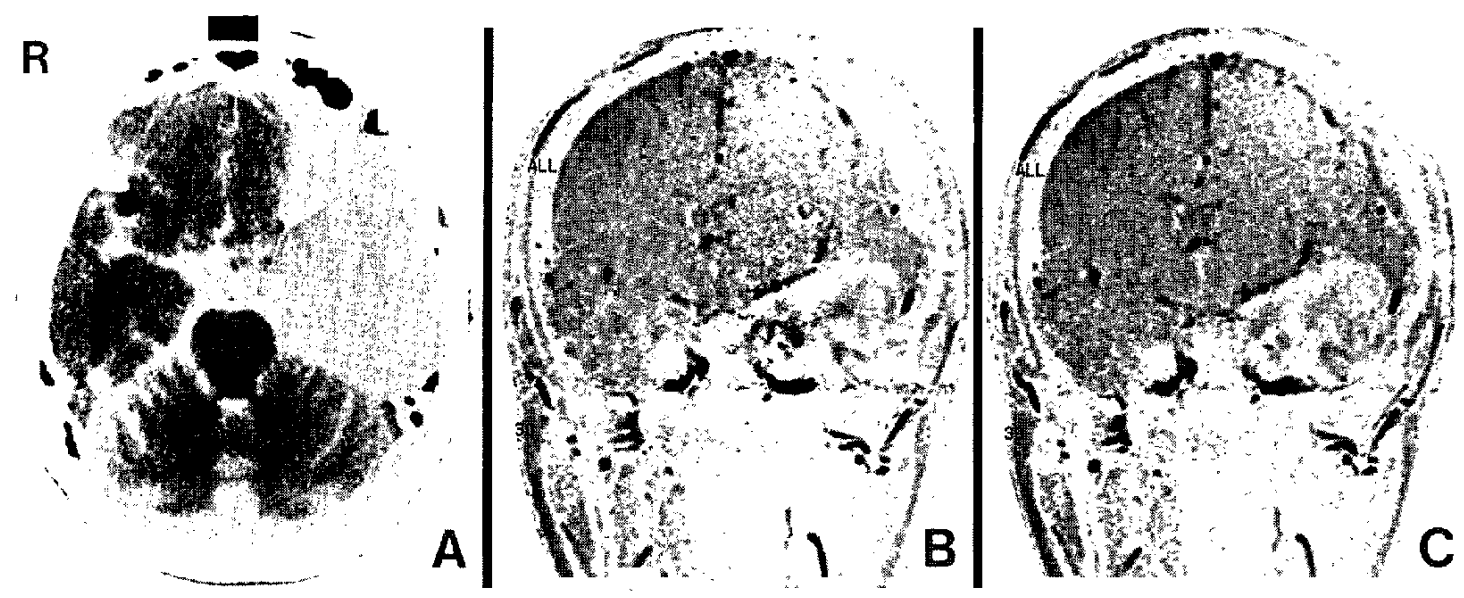

Fig. 6 Case 9 (postoperative study). CT cisternogram showing rapid filling (A). PC cine-MR images showing marked fluid motion within the cyst and jet flow between the cyst and suprasellar cistern $(\mathrm{B}, \mathrm{C})$.

8) Ohara S, Nagai H, Matsumoto T, Banno T: MR imaging of CSF pulsatory flow and its relation to intracranial pressure. $J$ Neurosurg 69: 675-682, 1988

9) Quencer RM, Donovan Post MJ, Hinks RS: CineMRI in the normal and abnormal CSF flow. $\mathrm{Neu}$ roradiology 32: 371-391, 1990

10) Sherman JL, Citrin CM: Magnetic resonance demonstration of normal CSF. AJNR Am J Neu- roradiol 7: 3-6, 1986

Address reprint requests to: T. Eguchi, M.D., Department of Neurosurgery, Osaka-Minami National Hospital, 677-2 Kido-cho, Kawachinagano, Osaka 586, Japan. 